\title{
Resultados y complicaciones de la endarterectomía carotídea en un Hospital de Madrid, España
}

\author{
Results and complications of carotid endarterectomy in a hospital from Madrid, Spain
}

\author{
Elena Martín-Morales ${ }^{1}$, Renato Jiménez-Román², AlexiaPaluso-Montero², TeresaHernández-Ruiz², \\ Covadonga Mendieta-Azcona ${ }^{2}$ y Luis F. Riera-del Moral** \\ ${ }^{1}$ Departamento de Cirugía, Facultad de Medicina, Universidad Autónoma de Madrid; ${ }^{2}$ Servicio de Angiología y Cirugía Vascular. Hospital Universitario \\ La Paz. Madrid, España
}

\begin{abstract}
Resumen
Objetivo: Conocer la incidencia de eventos cerebrovasculares y miocárdicos, y la supervivencia temprana, relacionados con la endarterectomía carotídea, y como objetivo secundario establecer los posibles factores de riesgo asociados a las complicaciones. Método: Estudio observacional de casos y controles anidado en una cohorte retrospectiva. Se incluyeron todos los pacientes que se sometieron a endarterectomía carotídea en el servicio de angiología y cirugía vascular del Hospital Universitario La Paz, de Madrid (España), en el periodo de enero de 2011 a diciembre de 2017. Para la estimación de diferencias se utilizó la prueba de ji al cuadrado. El análisis de supervivencia y permeabilidad se realizó mediante Kaplan-Meier y regresión de Cox. Resultados: Se realizaron 111 procedimientos en 108 pacientes, 95 (87.9\%) de ellos varones, con una edad media de $68.5 \pm 8.75$ años. La media de seguimiento fue de 2.9 años. No hubo mortalidad posquirúrgica a 30 días, y la tasa global de eventos vasculares cerebrales posoperatorios a 30 días fue del $2.7 \%$. Se encontró asociación entre la presencia de eventos vasculares cerebrales posquirúrgicos a 30 días y el cierre arterial primario $(p=0.005)$, y del infarto agudo de miocardio posoperatorio a 30 días y la reestenosis carotídea con el hábito tabáquico $(p=0.036$ y $p=0.008$, respectivamente). En el seguimiento a mediano plazo se encontró una tasa de enfermedad vascular cerebral y de infarto agudo de miocardio del 1,8\%. Conclusión: La endarterectomía carotídea es el procedimiento de elección en la estenosis carotídea por enfermedad aterosclerótica. En nuestro estudio se demuestran sus bajas tasas de mortalidad, de morbilidad y de complicaciones perioperatorias.
\end{abstract}

PALABRAS CLAVE: Complicación posquirúrgica. Endarterectomía carotídea. Estenosis carotídea. Ictus. Infarto de miocardio. Mortalidad.

\section{Abstract}

Objective: To study the incidence of cerebrovascular (transient ischemic attacks and stroke) and myocardial events (myocardial infarction) as well as early survival related to carotid endarterectomy. Our secondary aim is to establish possible risk factors associated with complications. Method: Retrospective observational case-control study within a cohort. All patients who underwent carotid endarterectomy by the angiology and vascular surgery service at the Hospital Universitario La Paz, in Madrid (Spain), in the period between January 2011 and December 2017 were included. Chi square was used to calculate differences. Kaplan-Meier and Cox regression was used for the survival analysis and patency. Results: 111 procedures were performed on 108 patients, $95(87,9 \%)$ male with an average age of $68.5 \pm 8.75$. The mean time of follow-up was 2.9 years. There was no 30-day post-surgical mortality, with a 30-day postoperative cerebral vascular event rate of $2.7 \%$. Statistically significant correlation was found between the presence of 30-day postoperative cerebral vascular event and primary closure $(p=0.005)$ 
as well as between the smoking habit and 30-day postoperative myocardial infarction $(p=0.036)$ and restenosis $(p=0.008)$. In mid-term follow-up, the event rate for cerebral vascular events and myocardial infarction was $1.8 \%$. Conclusion: carotid endarterectomy is the procedure of choice in carotid stenosis. The low rates of perioperative mortality, morbidity and complications have been demonstrated.

KEY WORDS: Complication, postoperative. Endarterectomy, carotid. Carotid stenosis. Stroke. Myocardial infarction. Mortality.

\section{Introducción}

La enfermedad vascular cerebral es la segunda causa de muerte, después de la cardiopatía isquémica, y la primera causa de incapacidad física en el adulto, en todo el mundo. Globalmente, la Organización Mundial de la Salud establece una incidencia de enfermedad vascular cerebral de 250 casos por 100.000 habitantes/año'. En España, según los datos de la Encuesta de Morbilidad Hospitalaria del Instituto Nacional de Estadística del año 2016, se registraron 119,659 casos de enfermedad vascular cerebral, lo que equivale a una incidencia de 257 episodios por cada 100.000 habitantes $^{2}$. Esta patología supone un gasto sociosanitario muy elevado, y se estima que va a incrementarse en los próximos años como consecuencia del envejecimiento de nuestra población.

Entre el 80 y el $85 \%$ de los eventos cerebrovasculares son de origen isquémico, mientras que del $10 \mathrm{al}$ $15 \%$ restante son hemorrágicos. Con respecto a los eventos neuroisquémicos, la mayoría son de etiología ateromatosa, tanto intracraneal como extracraneal ${ }^{3}$. La patología vascular extracraneal, principalmente la estenosis carotídea, es la causa de un 15 a un 50\% de los casos de enfermedad vascular cerebral de origen isquémico, ya sean accidentes isquémicos transitorios o infartos establecidos (ictus).

En los últimos años se han publicado numerosos estudios y guías de práctica clínica sobre la enfermedad arteriosclerosa extracraneal. Para prevenir nuevos eventos en pacientes con estenosis de arteria carótida extracraneal existen tres estrategias: el tratamiento médico solo, el tratamiento médico acompañando a la endarterectomía carotídea (CEA) o la colocación de un stent carotídeo. Todavía no se dispone de resultados definitivos de estudios que comparen los tres tipos de tratamiento, pero sí hay varios estudios internacionales muy importantes que comparan la colocación de un stent carotídeo y la CEA 4 .

La CEA es el procedimiento quirúrgico más estudiado y el tipo de intervención quirúrgica sobre la que más se ha publicado en la literatura científica ${ }^{5-9}$. Debido a ello y a sus particularidades intrínsecas, se trata de un procedimiento que debe ser auditado y revisado escrupulosamente por los equipos quirúrgicos que ofrecen este tratamiento en su práctica quirúrgica habitual.

A pesar de todos estos estudios, todavía es necesaria la documentación de nuevas guías de manejo práctico que arrojen mayor claridad y consistencia en las recomendaciones basadas en la evidencia científica ${ }^{5}$.

Para conocer la aplicabilidad de esos resultados en nuestro entorno y en la práctica clínica real, necesitamos saber las cifras de los resultados y las complicaciones que tenemos, de forma que podamos aplicar mejor y de manera más exacta las enseñanzas de artículos publicados en otros países, y que, por ende, nuestros pacientes sean tratados del mejor modo posible.

Con este estudio se busca demostrar que la aplicabilidad de la CEA concuerda con la observada en la literatura documentada, y evaluar cuáles son las complicaciones más frecuentes y graves, en qué condiciones se dan y qué se puede hacer para evitarlas. Su otro objetivo es analizar la posible asociación entre la aparición de complicaciones y diferentes factores de riesgo que presenten los pacientes o el proceso intraoperatorio.

\section{Método}

Se diseñó un estudio observacional de casos y controles anidado en una cohorte retrospectiva de pacientes tratados mediante CEA en el Hospital Universitario La Paz (Madrid, España), de manera consecutiva, entre enero de 2011 y diciembre de 2017. Los procedimientos estuvieron a cargo de ocho cirujanos especialistas en cirugía vascular.

\section{Definición de población del estudio}

Se incluyeron todos los pacientes intervenidos de CEA de forma electiva en el servicio de angiología y cirugía vascular del Hospital Universitario La Paz. Se excluyeron aquellos intervenidos de forma urgente, los intervenidos por traumatismos de la arteria carótida, por aneurismas o pseudoaneurismas carotídeos, o con 
cualquier otra patología diferente de la enfermedad oclusiva crónica de la arteria carótida extracraneal.

Los datos se obtuvieron de las historias clínicas de los pacientes, de forma retrospectiva, fundamentalmente del informe de valoración anestésica preoperatoria, del informe de alta, de los estudios radiológicos realizados y de las analíticas preoperatorias y posoperatorias.

Se tuvieron en cuenta las variables edad, sexo y factores de riesgo cardiovascular, como hipertensión arterial, diabetes mellitus, hábito tabáquico y dislipidemia. También se estudiaron el uso de puenteo vascular transitorio (shunt) intracarotídeo, la colocación de un parche en el sitio de endarterectomía y la presencia de complicaciones inmediatas (mortalidad precoz, síntomas neurológicos, coronarios o de nervios periféricos, infección de la herida quirúrgica) o tardías (reestenosis carotídea posendarterectomía).

\section{Análisis estadístico de los resultados}

Los resultados de frecuencia están expresados en términos absolutos, como porcentajes e intervalos de confianza. Las variables continuas están expresadas como media ( \pm desviación estándar) y mediana (rango) según la prueba de normalidad (test de Kolmogórov-Smirnov). Para la estimación de diferencias entre variables se utilizó la prueba de ji al cuadrado. El análisis de supervivencia y permeabilidad se realizó mediante Kaplan-Meier y regresión de Cox para establecer los factores asociados con la supervivencia o la permeabilidad en el seguimiento. Los datos se analizan usando el software de análisis estadístico SPSS (IBM Corp. Released 2011. IBM SPSS Statistics for Windows, Version 20.0. Armonk, NY: IBM Corp.).

Este estudio contó con la aprobación del Comité Ético de Investigación Clínica del Hospital Universitario La Paz y de la Universidad Autónoma de Madrid.

\section{Resultados}

Durante los 7 años del periodo de estudio se realizaron 113 CEA. Del análisis se han excluido dos procedimientos realizados sobre un mismo paciente, perdido en el seguimiento.

Se analizaron 111 procedimientos realizados en 108 pacientes, 95 hombres (87.9\%) y 13 mujeres (12.1\%). Las edades fluctuaron entre 47 y 85 años, con una media de $68.5 \pm 8.75$ años.

En cuanto a los factores de riesgo presentes, se encontraron hipertensión arterial en 89 (80.2\%),
Tabla 1. Factores de riesgo cardiovascular y características clínicas de los pacientes

\begin{tabular}{llc}
\hline & $\mathbf{n}$ & $\%$ \\
\hline Hipertensión arterial & 89 & 80.2 \\
Tabaquismo activo & 27 & 24.3 \\
Extabaquismo & 58 & 52.3 \\
Dislipidemia & 91 & 82.0 \\
Diabetes mellitus & 30 & 27.0 \\
Sintomáticas & 92 & 82.9 \\
Estenosis contralateral & 37 & 33.3 \\
Estenosis sintomáticas & 92 & 82.9 \\
Estenosis contralateral & 37 & 33.3 \\
\hline
\end{tabular}

tabaquismo activo en 27 (24.3\%), extabaquismo en $58(52.3 \%)$, dislipidemia en 91 (82.0\%), diabetes mellitus dependiente de insulina en $18(16.2 \%)$ y diabetes mellitus no dependiente de insulina en $12(10.8 \%)$. La mayoría de los pacientes tenían más de un factor de riesgo (Tabla 1).

Respecto a las manifestaciones clínicas, el $82.9 \%$ de los pacientes presentó varios tipos de ellas: isquemia cerebral transitoria, hemiparesia, disfasia, amaurosis fugax e infarto retiniano. Es de destacar que muchos pacientes tenían síntomas combinados. Diecinueve pacientes estaban asintomáticos (17.1\%), pero se indicó cirugía en ellos por presentar progresión de la enfermedad aterosclerótica durante el seguimiento ecográfico de una placa carotídea, cuando el porcentaje de estenosis carotídea superaba el $70 \%$ según criterios hemodinámicos.

En cuanto a la técnica quirúrgica, se utilizó una derivación temporal (shunt) intracarotídea en 5 (4.5\%) pacientes (Pruitt $\mathrm{F}^{\circledR}{ }^{\circledR}$ Carotid Shunt, LeMaitre ${ }^{\circledR}$ Vascular). Se realizó cierre con parche de dacrón en 107 (96.4\%) y cierre primario de la CEA en 4 (3.6\%); en estos cuatro casos se decidió realizar cierre directo porque las placas ateroscleróticas afectaban escasamente la arteria carótida interna y esta tenía un diámetro que, a criterio del cirujano, no permitiría que se produjera una estenosis tras la sutura directa y más bien podría adquirir un diámetro excesivo si el cierre se realizaba con parche.

\section{Complicaciones posoperatorias}

Definimos las complicaciones tempranas como aquellas acontecidas en los primeros 30 días del posoperatorios. Se produjeron $3(2.7 \%)$ eventos 
vasculares cerebrales en tres pacientes distintos, $2(1.8 \%)$ infartos agudos de miocardio en dos pacientes y $2(1.8 \%)$ episodios de crisis hipertensivas en otros dos pacientes. No hubo ninguna muerte en el posoperatorio inmediato (Tabla 2).

Los tres eventos vasculares cerebrales ocurrieron en pacientes que previamente habían mostrado síntomas de estenosis carotídea significativa (ya fuera por accidentes isquémicos transitorios de repetición o por infartos agudos). Todos ellos fueron infartos agudos objetivados por técnicas de imagen, dos en el periodo de reanimación posquirúrgico y el restante en el cuarto día del posoperatorio. Se consideró que uno de los infartos, al despertar de la anestesia, fue de etiología hemodinámica, puesto que el paciente presentó hipotensión intraoperatoria. El segundo infarto, al salir de la anestesia, fue de etiología dudosa (hemodinámica o embólica). En el paciente que padeció el ictus en el cuarto día del posoperatorio, la etiología más probable es la embólica. De los 19 pacientes asintomáticos, ninguno presentó enfermedad vascular cerebral en el posoperatorio.

De los dos pacientes que presentaron infarto agudo de miocardio posoperatorio, uno era previamente asintomático y se vio afectado por un síndrome coronario agudo sin elevación del segmento ST no $Q$, no complicado, durante el periodo de reanimación. El otro paciente, que había sido sintomático, presentó en el tercer día del posoperatorio una angina inestable y se le realizo un cateterismo cardiaco para identificar la arteria ocluida y tratarla.

En cuanto a las crisis hipertensivas, un paciente presentó sintomatología y el otro no la presentó.

\section{Complicaciones locales}

Se describió neuropatía periférica en 20 pacientes (18.0\%), definida como lesión de los pares craneales facial o hipogloso, o de alguno de sus ramos. De todas ellas, $18(90.0 \%)$ fueron de tipo transitorio y con recuperación completa en el posoperatorio. Dos casos fueron en pacientes intervenidos sin haber presentado sintomatología de estenosis carotídea. Seis (5.4\%) pacientes desarrollaron un hematoma en la herida quirúrgica, pero ninguno de ellos requirió reintervención. La disfagia o disfonía por lesión de ramos del nervio vago (nervio laríngeo recurrente) aconteció en 2 (1.8\%) pacientes, y ambos casos se resolvieron sin mayor complicación. Hubo una infección de la herida quirúrgica, un síndrome de hiperperfusión cerebral (resuelto durante el ingreso) y una tromboembolia pulmonar en un paciente que requirió reimplante de la arteria
Tabla 2. Complicaciones posoperatorias relacionadas con el procedimiento

\begin{tabular}{lcc}
\hline & $\mathbf{n}$ & $\%$ \\
\hline Evento cerebrovascular & 3 & 2.7 \\
Infarto agudo de miocardio & 2 & 1.8 \\
Crisis hipertensiva & 2 & 1.8 \\
Neuropatía periférica & 20 & 18.0 \\
Hematoma & 6 & 5.4 \\
Disfagia/disfonía & 2 & 1.8 \\
Infección de la herida & 1 & 0.9 \\
Tromboembolia pulmonar & 1 & 0.9 \\
\hline
\end{tabular}

carótida interna con trombectomía de esta en el posoperatorio inmediato de la CEA (Tabla 2).

En resumen, un $6.5 \%$ de las complicaciones tempranas ocurrieron en pacientes asintomáticos, frente al $93.5 \%$ que ocurrieron en pacientes previamente sintomáticos.

\section{Complicaciones tardías}

Se encontraron tres reestenosis carotídeas de más del $50 \%$ en el seguimiento mediante ecografía Doppler, de las cuales dos fueron reintervenidas mediante stenting carotídeo. Se evidenciaron 37 (33.33\%) estenosis contralaterales en el seguimiento, de las cuales tres fueron intervenidas en el periodo del estudio.

\section{Mortalidad}

No hubo mortalidad en los primeros 30 días posoperatorios. Se produjeron seis muertes en el seguimiento a largo plazo: cuatro por cáncer, una por insuficiencia respiratoria y una por hemorragia cerebral secundaria a traumatismo craneoencefálico.

\section{Contraste de hipótesis}

Se efectuó la prueba de ji al cuadrado, así como la prueba exacta de Fisher y análisis multivariante para cada una de las complicaciones, con el fin de determinar su relación con los factores de riesgo cardiovascular. Encontramos que la variable cierre primario (en lugar de cierre con parche de dacrón) se correlacionó de manera estadísticamente significativa $(p=0.005)$ con la presencia de eventos vasculares cerebrales posquirúrgicos. También se encontró que el hábito tabáquico se correlacionó de manera 
Tabla 3. Relación entre distintos cierres, uso de shunt, características clínicas y desarrollo de eventos cerebrovasculares posoperatorios

\begin{tabular}{lccc}
\hline & Eventos cerebrovasculares posoperatorios & Odds ratio (Intervalo de Confianza) & $\mathbf{p}$ \\
\hline Parche de dacrón & 2 & $0.06(0.00-0.82)$ & 0.005 \\
Cierre primario & 1 & & 0.596 \\
Shunt & 0 & & - \\
No shunt & 3 & & 0.566 \\
Sintomático & 3 & & 0.232 \\
Asintomático & 0 & $4.17(0.37-47.58)$ & \\
Estenosis contralateral & 2 & & \\
No estenosis contralateral & 1 & & \\
\hline
\end{tabular}

estadísticamente significativa con el desarrollo de infarto agudo de miocardio posoperatorio $(p=0.036)$ y con la reestenosis carotídea en el seguimiento a largo plazo $(p=0.008)$ (Tablas 3 a 5$)$.

\section{Discusión}

La enfermedad aterosclerótica extracraneal de las arterias carótidas ha sido ampliamente estudiada desde múltiples perspectivas. Es bien conocido que hay un vasto espectro de manifestaciones clínicas, desde pacientes completamente asintomáticos hasta eventos cerebrovasculares incapacitantes permanentes.

En la actualidad, la evolución natural de los eventos cerebrovasculares es bien conocida. Las guías de práctica clínica de la American Heart Association y de la European Society for Vascular Surgery (ESVS) apoyan que los pacientes con estenosis carotídea superior al $60 \%$ (asintomáticos) o al 50\% (aquellos que habían presentado sintomatología previa) se benefician de la CEA, puesto que el riesgo de recurrencia de ictus es de hasta el $50 \%$ en 5 años ${ }^{10,11}$. En aquellos con accidentes isquémicos transitorios previos, el riesgo de ictus es de un $35-50 \%$ a los 5 años, y el riesgo de incidencia de eventos neuroisquémicos está elevado en los pacientes asintomáticos ${ }^{12,13}$. El Asymptomatic Carotid Atheroesclerosis Study (ACAS) y otros estudios incluidos en un metaanálisis han mostrado que el efecto beneficioso de la CEA en pacientes asintomáticos con estenosis carotídeas graves $(>60 \%)$ se obtiene cuando el riesgo quirúrgico es menor del $3 \%{ }^{5}$; las guías de la ESVS publicadas en 2018 insisten en estas recomendaciones. Por lo tanto, se establece la indicación quirúrgica en pacientes asintomáticos siempre y cuando presenten una lesión estenosante grave y bajo riesgo operatorio. Los pacientes asintomáticos intervenidos en este estudio fueron el $17.1 \%$, un porcentaje
Tabla 4. Relación entre factores de riesgo cardiovasculares y desarrollo de infarto agudo de miocardio posquirúrgico

\begin{tabular}{lcc}
\hline & $\begin{array}{c}\text { Infarto agudo de miocardio } \\
\text { posoperatorio }\end{array}$ & $\mathbf{p}$ \\
\hline Fumador activo & 2 & 0.036 \\
No fumador & 0 & \\
Hipertensión arterial & 2 & 0.641 \\
No hipertensión arterial & 0 & \\
\hline
\end{tabular}

Tabla 5. Relación entre factores de riesgo cardiovasculares y reestenosis carotídea

\begin{tabular}{lccc}
\hline & Reestenosis & $\begin{array}{c}\text { Odds ratio } \\
\text { (Intervalo de Confianza) }\end{array}$ & $\mathbf{p}$ \\
\hline Fumador activo & 3 & 0.008 \\
No fumador & 0 & $0.48(0.04-5.58)$ & 0.488 \\
Hipertensión arterial & 2 & & \\
No hipertensión arterial & 1 & & 0.895 \\
Parche de dacrón & 3 & & \\
Cierre primario & 0 & & \\
\hline
\end{tabular}

similar al de los estudios de otros autores. A la vista de nuestros resultados (tasa de eventos neurológicos postoperatorios del $2.7 \%$ ), nuestro grupo puede y debe ofrecer la CEA a este tipo de pacientes.

La CEA es una intervención con una larga trayectoria histórica ${ }^{14}$ y se realiza de manera habitual en los servicios de cirugía vascular de todo el mundo. A pesar de ello, es necesario que las guías de práctica clínica aporten de manera integral las condiciones y las evaluaciones que deban llevarse a cabo en los candidatos a cirugía, con el fin de individualizar el tratamiento que corresponda a los factores de riesgo y al grado de lesión de cada paciente. 
En cuanto a las técnicas quirúrgicas, la técnica convencional se inicia con una incisión longitudinal anterior al músculo esternocleidomastoideo y mediante un abordaje anterior a la vena yugular se accede a la arteria carótida, para luego, mediante una incisión longitudinal desde la arteria carótida común hasta la interna, seccionar y extraer la placa de ateroma, con previo clampaje del trípode carotídeo y heparinización sistémica. En nuestro caso, la técnica quirúrgica utilizada fue la estándar. La última guía de la ESVS deja a criterio del cirujano el tipo de incisión, así como optar por un abordaje anterior o retroyugular (recomendación IB). También deja a criterio del cirujano optar por una técnica distinta, mediante eversión de la arteria carótida (recomendación IA).

Todos los pacientes fueron intervenidos bajo anestesia general. Algunos cirujanos vasculares afirman que la anestesia regional tiene mejores resultados, ya que permite detectar cualquier problema neurológico de manera más rápida durante el clampaje de las carótidas, y lo realizan de manera sistemática. Hasta el día de hoy no se han podido demostrar diferencias entre el uso de anestesia general o regional en la CEA, y las guías actuales dejan a criterio del equipo quirúrgico la elección de la técnica ${ }^{9,15}$.

En cuanto al tipo de cierre, a la práctica totalidad de nuestros pacientes se les realizó un cierre con parche de dacrón. Las últimas guías recomiendan un cierre con parche sobre el cierre mediante sutura directa, sin indicar preferencia sobre el material (parche de vena, dacrón, Politetrafluoroetileno expandido (PTFE), pericardio) (recomendación IA). En este estudio encontramos que los pacientes a quienes se les realizó un cierre directo presentaron un aumento en el riesgo de eventos vasculares cerebrales posquirúrgicos.

Con respecto al uso de derivación temporal (shunts), nuestro grupo opta por una modalidad selectiva, utilizándolos solo cuando se aprecian cambios en la monitorización de la actividad cerebral (electroencefalograma, potenciales evocados). Las recomendaciones actuales dejan, nuevamente, la decisión de su uso al cirujano22.

Tomando como referencia las tasas de mortalidad y morbilidad neurológica del informe del Stroke Council of American Heart Association y la ESVS, vemos que las cifras aceptadas son un 3\% en asintomáticos, un $5 \%$ en pacientes con accidentes isquémicos transitorios y un $7 \%$ en pacientes con ictus previos ${ }^{6}$. En nuestro estudio, la tasa de morbimortalidad total es del $2.7 \%$ (mortalidad perioperatoria del $0 \%$ y eventos cerebrovasculares en el 2.7\%). Estos resultados están en consonancia con los obtenidos en los estudios multicéntricos que demostraban que la cirugía superaba al tratamiento médico en pacientes sintomáticos con estenosis carotídea, con tasas de morbimortalidad del $5.8 \%$ y el $7.5 \%$, respectivamente ${ }^{16,17}$. La tasa de mortalidad perioperatoria varía entre el $0.5 \%$ y $1.8 \%$, y de acuerdo con los ensayos con mayores muestras de CEA, la causa más frecuente es el infarto agudo de miocardio. Las muertes secundarias a eventos neuroisquémicos suponen entre un $0 \%$ y un $0.6 \%$, según la literatura ${ }^{6,16}$.

En cuanto a la lesión de los nervios craneales, en la literatura alcanza cifras de hasta el 17\%, con disfunción del hipogloso, el ramo mandibular del facial y el nervio laríngeo recurrente como lesiones más comunes ${ }^{18}$. En nuestro estudio observamos un $18 \%$ de daño en los nervios hipogloso y facial, y un $1.8 \%$ en el nervio laríngeo recurrente. La mayor parte de las lesiones de ramos o de nervios son reversibles, como ocurrió en nuestros pacientes, aunque hubo dos casos de lesiones persistentes.

En cuanto a complicaciones menos frecuentes, la infección de la herida quirúrgica solo ocurrió en un paciente, y otro presentó síndrome de hiperperfusión; los dos casos se resolvieron y ambas complicaciones entran dentro de lo esperable según la literatura ${ }^{18}$.

La estenosis carotídea contralateral es una situación muy común, que se presenta hasta en el $80 \%$ de los pacientes ${ }^{6}$. Nuestra tasa de estenosis contralateral posterior a la CEA es del $33.33 \%$. En cuanto a la intervención sobre las estenosis contralaterales, las guías no aconsejan una intervención simultánea. Al realizarse en dos tiempos, algunos abogan por que la intervención debe realizarse 2 días después de la CEA contralateral, mientras que otros prefieren esperar 2 semanas y otros diferir la cirugía y estudiar la estenosis con las mismas técnicas y criterios que la anterior ${ }^{19}$. Nuestro grupo es partidario de esta última pauta, de acuerdo con un abordaje multidisciplinario en el que participan también neurólogos y neurorradiólogos.

La reestenosis significativa (más del $50 \%$ de oclusión) ocurre el $1 \%$ al $20 \%$ de los casos, y es necesaria la reintervención en el $1 \%$ al $3 \%$ de ellas ${ }^{6}$. Aplicado a nuestro estudio, hubo tres pacientes que presentaron reestenosis significativa, lo que corresponde a un $2.7 \%$; sin embargo, solo $2(1,8 \%)$ precisaron reintervención, pues fueron sintomáticos, y se realizó mediante el implante de un stent carotídeo. Las recomendaciones actuales indican que las reestenosis sintomáticas de un 50-99\% deben ser intervenidas, ya sea mediante una nueva CEA o mediante el implante de un stent carotídeo.

Por último, es destacable que se halló asociación para algunas de las variables estudiadas: la plastia mediante 
cierre primario comparada con el cierre con parche de dacrón aumenta el riesgo de evento vascular cerebral posquirúrgico; el hábito tabáquico, por otra parte, aumenta la tasa de infarto agudo de miocardio posoperatorio $(p=0.036)$ y de reestenosis carotídea $(p=0.008)$. A partir de estas correlaciones, que concuerdan con las halladas en otros estudios $20,21,22$, podemos aseverar que la plastia con cierre mediante parche de dacrón y el no fumar pueden disminuir el riesgo de complicaciones posquirúrgicas en los pacientes sometidos a CEA.

El objetivo ahora es seguir comparando la CEA con las nuevas tecnologías de cirugía endovascular, que están en auge y auguran un futuro prometedor en las próximas décadas.

\section{Conclusión}

La CEA es el procedimiento de elección en la estenosis carotídea. En nuestro estudio se demuestran sus bajas tasas de mortalidad, morbilidad y complicaciones perioperatorias, así como el excelente pronóstico del procedimiento. En este estudio, los resultados obtenidos son comparables con los de estudios internacionales que sirven de referencia para la práctica clínica.

\section{Agradecimientos}

A todos los integrantes del servicio de angiología y cirugía vascular del Hospital Universitario La Paz de Madrid, adjuntos, residentes, enfermeras y técnicos.

\section{Conflicto de intereses}

Los autores declaran que no existe ningún conflicto de intereses.

\section{Financiamiento}

Este estudio no ha recibido financiamiento externo.

\section{Responsabilidades éticas}

Protección de personas y animales. Los autores declaran que para esta investigación no se han realizado experimentos en seres humanos ni en animales.

Confidencialidad de los datos. Los autores declaran que han seguido los protocolos de su centro de trabajo sobre la publicación de datos de pacientes.

Derecho a la privacidad y consentimiento informado. Los autores han obtenido el consentimiento informado de los pacientes y/o sujetos referidos en el artículo. Este documento obra en poder del autor de correspondencia.

\section{Bibliografía}

1. Benjamin EJ, Blaha MJ, Chiuve SE, Cushman M, Das SR, Deo R, et al. Heart disease and stroke statistics' 2017 update: a report from the American Heart Association. Circulation. 2017; e146-e603.

2. Encuesta de morbilidad hospitalaria. Año 2016. Madrid: Instituto Nacional de Estadística; 2016. p. 1-10. Disponible en: http://www.ine.es/CDINEbase/consultar.do?mes=\&operacion=Encuesta+de+morbilidad+hospitalaria\&id_oper=Ir

3. Brainin M, Bornstein N, Boysen G, Demarin V. Acute neurological stroke care in Europe: results of the European Stroke Care Inventory. Eur J Neurol. 2000;7:5-10

4. Bartko D, Combor I, Pechanova O, Boselova M. Carotid endarterectomy (CEA), carotid artery stenting (CAS) and stroke recovery which of them are better for recovery of brain functions or prevention of cognitive decline? J Neurol Sci. 2009;283:244.

5. Abbott AL, Paraskevas KI, Kakkos SK, Golledge J, Eckstein $\mathrm{HH}$, Diaz-Sandoval LJ, et al. Systematic review of guidelines for the management of asymptomatic and symptomatic carotid stenosis. Stroke. 2015;46:3288-301.

6. Biller J, Feinberg WM, Castaldo JE, Whittemore AD, Harbaugh RE, Dempsey RJ, et al. Guidelines for carotid endarterectomy: a statement for healthcare professionals from a special writing group of the stroke council, American Heart Association. Circulation. 1998:97:501-9.

7. Hillbom M. What supports the role of alcohol as a risk factor for stroke? J Intern Med. 2009;221:93-106.

8. Wilson PWF, Garrison RJ, Castelli WP. Postmenopausal estrogen use, cigarette smoking and cardiovascular morbidity in women over 50: The Framingham study. Obstet Gynecol Surv. 1986;41:306-7.

9. Rockman CB, Riles TS, Gold M, Lamparello PJ, Giangola G, Adelman MA, et al. A comparison of regional and general anesthesia in patients undergoing carotid endarterectomy. J Vasc Surg. 1996;24:946-56.

10. Executive Committee for the Asymptomatic Carotid Atherosclerosis Study. Endarterectomy for asymptomatic carotid artery stenosis. JAMA. 1995;273:1421-8.

11. Inzitari D, Eliasziw M, Gates $P$, Sharpe BL, Chan RKT, Meldrum HE, et al.; N.A.S.C. The causes and risk of stroke in patients with asymptomatic internal-carotid-artery stenosis. N Engl J Med. 2000;342:1693-700.

12. Moneta G. Risk of early carotid endarterectomy for symptomatic carotid stenosis. Yearbook of Vascular Surgery. 2011; p. 243-4.

13. Liberato $B$, Rundek T. Risk stratification of patients with asymptomatic carotid artery stenosis: evaluation of carotid stenosis severity, progression and morphology by duplex ultrasound. En: Mousa I, Rundek T, editores. Asymptomatic carotid artery disease. New York: Taylor \& Francis; 2007. p. 107-17.

14. Eastcott HHG, Pickering GW, Rob CG. Reconstruction of internal carotid artery in a patient with intermittent attacks of hemiplegia. Lancet. 1954;264:994-6

15. Aboyans V, Ricco JB, Bartelink MEL, Björck M, Brodmann M, Cohnert T, et al. Editor's Choice - 2017 ESC Guidelines on the Diagnosis and Treatment of Peripheral Arterial Diseases, in collaboration with the European Society for Vascular Surgery (ESVS). Eur J Vasc Endovascular Surg. 2018;55:305-68.

16. Ricotta JJ, Aburahma A, Ascher E, Eskandari M, Faries P, Lal BK. Updated Society for Vascular Surgery guidelines for management of extracranial carotid disease. J Vasc Surg. 2011;54:e1-31.

17. Barnett HJ, Taylor DW, Eliasziw M, Fox AJ, Ferguson GG, Haynes RB, et al. Benefit of carotid endarterectomy in patients with symptomatic moderate or severe stenosis. North American Symptomatic Carotid Endarterectomy Trial Collaborators. N Engl J Med. 1998;339:1415-25.

18. Arnold M, Perler BA. Carotid Artery Endarterectomy. En: Cronenwett JL, Johnston KW, editores. Rutherford's Vascular Surgery. $8^{\text {th }}$ ed. Philadelphia: Elsevier; 2014. p. 1533-8.

19. Darling RC $3^{\text {rd }}$, Kubaska S, Shah DM, Paty PS, Chang BB, Lloyd WE et al. Bilateral carotid endarterectomy during the same hospital admission. Cardiovasc Surg. 1996;4:759-62.

20. AbuRahma AF, Hopkins ES, Robinson PA, Deel JT, Agarwal S, Smith RB, et al. Prospective randomized trial of carotid endarterectomy with polytetrafluoroethylene versus collagen-impregnated dacron $(\mathrm{He}-$ mashield) patching: late follow-up. Ann Surg. 2003;237:885-93.

21. Andrikopoulos V, Antoniou I, Papacharalambous G, Panousis P. Our experience with carotid endarterectomy without patch and shunt. Int Angiol. 1996;15:307-11.

22. Naylor AR, Ricco JB, de Borst GJ, Debus S, de Haro J, Halliday A, et al. Editor's Choice - Management of atherosclerotic carotid and vertebral artery disease: 2017 clinical practice guidelines of the European Society for Vascular Surgery (ESVS). Eur J Vasc Endovasc Surg. 2018;55:3-81. 